\title{
A simulation study with a new residual ionospheric error model for GPS radio occultation climatologies
}

\author{
J. Danzer ${ }^{1}$, S. B. Healy ${ }^{2}$, and I. D. Culverwell ${ }^{3}$ \\ ${ }^{1}$ Wegener Center for Climate and Global Change (WEGC), University of Graz, Graz, Austria \\ ${ }^{2}$ European Centre for Medium-range Weather Forecasts (ECMWF), Reading, UK \\ ${ }^{3}$ Met Office, Exeter, UK \\ Correspondence to: J. Danzer (julia.danzer@uni-graz.at)
}

Received: 23 October 2014 - Published in Atmos. Meas. Tech. Discuss.: 27 January 2015

Revised: 22 April 2015 - Accepted: 4 May 2015 - Published: 21 August 2015

\begin{abstract}
In this study, a new model was explored which corrects for higher order ionospheric residuals in Global Positioning System (GPS) radio occultation (RO) data. Recently, the theoretical basis of this new "residual ionospheric error model" has been outlined (Healy and Culverwell, 2015). The method was tested in simulations with a one-dimensional model ionosphere.

The proposed new model for computing the residual ionospheric error is the product of two factors, one of which expresses its variation from profile to profile and from time to time in terms of measurable quantities (the L1 and L2 bending angles), while the other describes the weak variation with altitude. A simple integral expression for the residual error (Vorob'ev and Krasil'nikova, 1994) has been shown to be in excellent numerical agreement with the exact value, for a simple Chapman layer ionosphere. In this case, the "altitudinal" element of the residual error varies (decreases) by no more than about $25 \%$ between $\sim 10$ and $\sim 100 \mathrm{~km}$ for physically reasonable Chapman layer parameters. For other simple model ionospheres the integral can be evaluated exactly, and results are in reasonable agreement with those of an equivalent Chapman layer.

In this follow-up study the overall objective was to explore the validity of the new residual ionospheric error model for more detailed simulations, based on modeling through a complex three-dimensional ionosphere.

The simulation study was set up, simulating day and night GPS RO profiles for the period of a solar cycle with and without an ionosphere. The residual ionospheric error was studied, the new error model was tested, and temporal and spatial variations of the model were investigated. The model per-
\end{abstract}

formed well in the simulation study, capturing the temporal variability of the ionospheric residual. Although it was not possible, due to high noise of the simulated bending-angle profiles at mid- to high latitudes, to perform a thorough latitudinal investigation of the performance of the model, first positive and encouraging results were found at low latitudes. Furthermore, first application tests of the model on the data showed a reduction in temperature level of the ionospheric residual at $40 \mathrm{~km}$ from about -2.2 to $-0.2 \mathrm{~K}$.

\section{Introduction}

The radio occultation (RO) technique gains information about the physical properties of a planetary atmosphere by detecting a change in a radio signal when it passes through this atmosphere. With the instalment of the Global Positioning System (GPS) constellation this principle could be applied to scan the Earth's atmosphere. Using the GPS frequencies $f_{1}(1575.42 \mathrm{MHz})$ and $f_{2}(1227.60 \mathrm{MHz})$, the RO technique has provided high-quality profiles in the upper troposphere and lower stratosphere (UTLS) since 1995 (see, e.g., Kursinski et al., 1997; Steiner et al., 2001; Hajj et al., 2002). It has the advantage of all-weather capability, high vertical resolution, and global coverage. RO data have significantly reduced systematic errors in global weather analysis (e.g., Healy and Thépaut, 2006; Cardinali and Healy, 2014) and are very useful for climate monitoring (e.g., Foelsche et al., 2009).

The measured observables during an $\mathrm{RO}$ event are the phase delays of the transmitted electromagnetic signals L1 
and L2, which are detected from a low Earth orbit (LEO) satellite. From the primary quantity of phase delay, bending angles and, after further processing, geophysical information such as temperature and pressure can be obtained. However, the total phase delay of the signals consists of neutral atmospheric phase delays as well as ionospheric phase delays. In order to be able to study the characteristics of the neutral atmosphere, the use of an ionospheric correction procedure is necessary. To first order it is possible to remove the ionospheric contribution (see, e.g., Spilker, 1980; Vorob'ev and Krasil'nikova, 1994; Ladreiter and Kirchengast, 1996; Syndergaard, 2000). Nevertheless, higher-order ionospheric residuals remain, which affect the climate monitoring capability especially in the stratosphere.

The remaining residual ionospheric error is a function which varies with the 11-year solar cycle, being higher at daytime compared to nighttime (Danzer et al., 2013). There is concern about the impact of this residual ionospheric error on GPS RO level-3 products $^{1}$ (Mannucci et al., 2011). The sensitivity of temperature level-3 products towards small bending-angle biases has been tested. Adding a bendingangle bias of $0.05 \mu \mathrm{rad}$ to an entire bending angle resulted in about a $0.5 \mathrm{~K}$ difference at $30 \mathrm{~km}$ altitude in temperature (e.g., studies by Rocken et al., 2008, 2009; Schreiner et al., 2011). For comparison, solar maximum and daytime conditions shows bending-angle biases of about $0.3 \mu \mathrm{rad}$, emphasizing the importance of a better understanding of residual ionospheric errors.

Residual ionospheric errors are often described as the omission of higher-order magnetic terms in the ionospheric refractive index. It has been noted by Healy and Culverwell (2015) that the research article by Vorob'ev and Krasil'nikova (1994) contains an integral expression (their Eq. 22), valid for a spherically symmetric ionosphere with no magnetic field. This is a mathematical correction to the usual Abel integral formula for the bending angle in terms of the refractivity, rather than a physical correction for neglected processes. The former turns out, however, to be a systematic correction, whereas the latter depends on the direction of the magnetic field. It is reasonable to expect, then, that in a climatological sense Vorob'ev and Krasil'nikova's expression would be the dominant one.

Healy and Culverwell made calculations based on this expression which showed that it produces residual errors comparable to those produced in more complex simulations. They also noticed that in this model there is a simple relationship between the residual error and the L1 and L2 bending angles $\alpha_{1}$ and $\alpha_{2}$, which allows the former to be written

\footnotetext{
${ }^{1}$ Radio Occultation Meteorology Satellite Applications Facility (ROM SAF) level-3 products describe the monthly mean state of the atmosphere in the form of zonal averages, i.e., averages over all longitudes in $5^{\circ}$ latitude bands. The range of data products includes both RO-specific variables (bending angle, refractivity) and common geophysical variables (temperature, humidity, pressure).
}

as the product of rapidly varying factor, which can be calculated from observable quantities, and a more slowly varying factor (in altitude and, potentially, time; see Sect. 2). The first factor depends on the square of the total electron content (TEC; i.e., the vertically integrated electron number density), as does the residual error itself. The second factor depends on the peak height and thickness of the ionosphere, which vary, more slowly, with the season and geographic location (see Eqs. 2-4). Preliminary results with idealized simulated bending angles showed reasonable agreement between theory and simulated observation, and this prompted the current study, which compares the theory to more realistic simulated radio occultation data.

The proposed new residual ionospheric error model is mainly suited for performing a bending-angle correction on bending-angle climatologies - i.e., it is understood as a climatological correction - instead of applying it on single bending-angle profiles. Recently, it has been proposed for climatological studies with GPS RO data to perform the averaging of the atmospheric parameters already in bending-angle space (Ao et al., 2012; Gleisner and Healy, 2013; Danzer et al., 2014). This avoids the usage of a priori information in the data through a complicated statistical optimization step, which was detected in the ROtrends study to be a major error source between the main processing centers of RO data (Ho et al., 2012; Steiner et al., 2013). In the newly introduced bending-angle averaging approach the observational bending-angle climatologies are used up to an altitude of $80 \mathrm{~km}$, which taking into account the influence of the ionosphere. The proposed residual ionospheric error model of this study would be highly suited for the bending-angle averaging approach.

The model does not correct for the residual ionospheric error that arises from horizontal gradients of the ionosphere, or those errors that are caused by the Earth's magnetic field (see companion paper Healy and Culverwell, 2015). These errors could have an effect on individual profiles ${ }^{2}$, but they should average out of the zonal monthly mean climatologies which are the focus of our study here.

In this specific follow-up study the work of Healy and Culverwell is extended, by testing the new residual ionospheric error model for a more complex situation, using simulated GPS RO data (see description of data, Sect. 3). RO events were simulated from 2001 until 2011, modeling according to the solar cycle a three-dimensional nonspherically symmetric ionosphere through which the L1 and L2 signals pass. Furthermore colocated neutral atmospheric profiles were simulated. Monthly-mean residual ionospheric errors were computed, testing its temporal as well as spatial variability. The key question was if the proposed error model captures the simulated residual error as a function of

\footnotetext{
${ }^{2}$ Although Syndergaard (2000) argued that the geomagnetic term has no appreciable impact on the residual ionospheric errors in GPS RO applications.
} 
time and space (Sect. 4.1). Furthermore, first tests of correcting the residual error on bending-angle level were performed, and its effect on temperature level was studied (Sect. 4.2).

The study should help us to say whether the residual ionospheric error model is applicable to the more complex situation of a three-dimensional non-spherically symmetric ionosphere.

\section{Residual ionospheric error model}

To first order the ionosphere-corrected bending angle $\alpha_{\mathrm{C}}$ is given by (using the formulation from Vorob'ev and Krasil'nikova, 1994)

$\alpha_{\mathrm{C}}(a)=\frac{f_{1}^{2} \alpha_{1}(a)-f_{2}^{2} \alpha_{2}(a)}{f_{1}^{2}-f_{2}^{2}}$,

where $\alpha_{1}$ and $\alpha_{2}$ are the $f_{1}$ and $f_{2}$ signal bending defined at the same impact parameter $a$ (which is the perpendicular distance between one of the ray asymptotes and the center of refraction).

It is known that the magnitude of the residual ionospheric errors corresponds to the ionospheric electron density values; i.e., larger electron densities produce larger residual errors. Vorob'ev and Krasil'nikova (1994) provide an integral expression for the residual ionospheric error $\Delta \alpha$, given for the case of a one-dimensional ionosphere with no magnetic field. (Liu et al. (2013) conclude that the magnetic field has no essential impact on bending-angle residuals.) This residual error depends on the vertical gradient of the square of the electron concentration $\left(n_{\mathrm{e}}^{2}\right)$, as follows ${ }^{3}$ :

$$
\begin{aligned}
\Delta \alpha(a) & \propto a \int_{a}^{\infty} \frac{\left(2 r^{2}-a^{2}\right)\left(\frac{\mathrm{d}\left(n_{\mathrm{e}}^{2}\right)}{\mathrm{d} r}\right)}{\left(r^{2}-a^{2}\right)^{3 / 2}} \mathrm{~d} r \\
& \propto \frac{\mathrm{TEC}^{2}}{H f^{4}} \times \text { some slowly varying function of } a \text { and } r_{0},
\end{aligned}
$$

where $r$ is the radial ray path coordinate, $H$ is some measure of the thickness of the ionosphere, and $r_{0}$ is the height of its peak. On the other hand, the ionospheric bending is related to the vertical integral of the gradient of electron density, as follows:

$$
\begin{aligned}
\alpha(a) & \propto a \int_{a}^{\infty} \frac{\left(\frac{\mathrm{d}\left(n_{\mathrm{e}}\right)}{\mathrm{d} r}\right)}{\left(r^{2}-a^{2}\right)^{1 / 2}} \mathrm{~d} r \\
& \propto \frac{\text { TEC }}{f^{2}} \times \text { another slowly varying function of } a \text { and } r_{0} .
\end{aligned}
$$

The last two equations suggest a relationship between the residual and the actual bending angle. Healy and Culverwell calculated the residual ionospheric error for the case of

\footnotetext{
${ }^{3}$ The numerator of VK94 Eq. (22) differs slightly, which has however no significant impact on the error estimate.
}

a Chapman layer ionosphere and found, as expected from the above, that the residual error $\Delta \alpha(a)$ as a function of impact parameter $a$ can be written

$\Delta \alpha(a) \equiv \alpha_{C}(a)-\alpha_{N}(a)=-\kappa(a)\left(\alpha_{1}(a)-\alpha_{2}(a)\right)^{2}$,

with $\alpha_{C}(a)$ being the first-order ionosphere-corrected bending angle (see Eq. 1) and $\alpha_{N}(a)$ being the neutral atmospheric bending angle. The residual ionospheric error in Eq. (4) is written as the product of two factors, as follows:

$$
\begin{array}{lll}
\alpha_{C}(a)-\alpha_{N}(a) & \ldots & \text { residual error, } \propto \mathrm{TEC}^{2} \\
\left(\alpha_{1}(a)-\alpha_{2}(a)\right)^{2} & \ldots & \begin{array}{l}
\text { rapidly varying factor, } \\
\text { slowly varying factor, } \\
\kappa(a)
\end{array} \\
& \ldots & \begin{array}{l}
2 \\
\text { independent of TEC. }
\end{array}
\end{array}
$$

(Differencing the L1 and L2 bending angles removes the neutral bending angle, which is the principal component of the bending below about $40 \mathrm{~km}$.) The simple expression in Eq. (4) for the residual error is the key formula in this study. $\kappa(a)$ can be written as a relatively simple function of the impact parameter, the peak height of the Chapman layer, and its width (Healy and Culverwell, 2015). These authors calculated the bending and its residual for a one-dimensional Chapman layer and showed that $\kappa(a)$ was indeed a slowly varying function of impact altitude (defined as impact parameter minus radius of curvature minus geoid undulation). For example, for a Chapman layer of width $H=\sim 75 \mathrm{~km}$ and peak height $r_{0}=\sim 300 \mathrm{~km}, \kappa$ decreases monotonically from about $16 \mathrm{rad}^{-1}$ at $\sim 10 \mathrm{~km}$ to about $12 \mathrm{rad}^{-1}$ at $\sim 100 \mathrm{~km}$. For a range of realistic $r_{0} \mathrm{~s}$ and $H \mathrm{~s}, \kappa$ varies by less than a factor of 2 at any given height between $\sim 10$ and $\sim 100 \mathrm{~km}$.

We suggest that the new model term (Eq. 4) might be important for producing climatologies, if the $\left(\alpha_{1}(a)-\alpha_{2}(a)\right)^{2}$ factor captures the main temporal variability of the residual error. Given the potential improvements on GPS RO level-3 data products that might arise from the application of Eq. (4), it is clear that the model needs to be investigated for more realistic data and more complicated situations. Such a study is the focus of the current paper.

We perform a simulation study, since this has the decisive advantage that the residual error $\Delta \alpha(a)$ can be calculated directly. RO events were simulated with and without a model ionosphere, making it possible to calculate the difference between the ionosphere-corrected bending angle $\alpha_{C}(a)$ (simulations with ionosphere) and the neutral bending angle $\alpha_{N}(a)$ (simulations without ionosphere) at same time and space. Calculating also the $\left(\alpha_{1}(a)-\alpha_{2}(a)\right)^{2}$ factor enables us to study the coefficient $\kappa(a)$, dependent on impact parameter $a$, space (latitudinal dependence), and any time dependence not appearing in the bending-angle differences themselves (Sect. 4.1). If the $\left(\alpha_{1}(a)-\alpha_{2}(a)\right)^{2}$ term in the proposed model for the residual ionospheric error (Eq. 4) captures the rapid temporal variability of the residual error, then $\kappa(a)$ should be almost constant with time. After $\kappa(a)$ has been assessed, a first bending-angle correction using the residual error model can be performed (see Sect. 4.2). For 
the correction of the bending-angle profiles, Eq. (4) is simply rewritten, and the neutral atmospheric bending angle is found by

$\alpha_{N}(a)=\alpha_{C}(a)+\kappa(a)\left(\alpha_{1}(a)-\alpha_{2}(a)\right)^{2}$,

which we call from now on the "residual ionospheric error correction" (RESIC).

The advantage of the RESIC model is that the rapidly varying component of the residual error can be directly calculated from the GPS RO data. Hence the solar cycle dependency can be captured quite easily. However, the coefficient $\kappa(a)$ from the model needs to be determined, either from simulation studies or theoretical models, before Eq. (6) can be applied to real observational bending-angle climatologies.

\section{Data sets}

With the EGOPS software (End-to-End Generic Occultation Performance and Processing System) version 5.5 (Fritzer et al., 2009) we performed an end-to-end simulation study, simulating daytime (12:00 and 15:00 LT) and nighttime (02:00 LT) profiles, similar to the study in Danzer et al. (2013). The profiles were simulated for the years 2001 to 2011 via ray tracing through ionospheric and neutral atmospheric fields. During the simulations the neutral atmosphere was held constant, while the ionosphere was varied for each profile according to the solar cycle in this period of time. For the constant neutral atmosphere we used an operational analysis field provided by the ECMWF (European Centre for Medium-Range Weather Forecasts) from 1 January 2007 (i.e., in the middle of the period under discussion) at T42L91 resolution. The horizontal resolution T42 corresponds to the resolution of RO data $(300 \mathrm{~km})$, with data available at 91 vertical levels (L91).

The non-spherical three-dimensional ionosphere was simulated with the NeUoG model from the University of Graz (Leitinger et al., 1995; Leitinger and Kirchengast, 1997), which is driven by the $F_{10.7}$ index as an indicator of the solar activity. The $F_{10.7}$ index is based on the solar radio flux at a wavelength of $10.7 \mathrm{~cm}$ and is given in terms of sfu (solar flux unit), where $1 \mathrm{sfu}=10^{-22} \mathrm{~W} \mathrm{~m}^{-2} \mathrm{~Hz}^{-1}$. The $F_{10.7}$ data, given on a daily basis, were downloaded from the website of the National Oceanic and Atmospheric Administration; see NOAA (2012). Figure 1 shows the $F_{10.7}$ data on a monthly basis for the years 2001 until 2011. We produced events taking place in all Januaries from 2001 to 2011 at latitude bands $0,5^{\circ} \mathrm{S} / \mathrm{N}, 10^{\circ} \mathrm{S} / \mathrm{N}$, continuing in $10^{\circ}$ steps until $60^{\circ} \mathrm{S} / \mathrm{N}$. Each latitude within $10^{\circ} \mathrm{S}-\mathrm{N}$ was simulated at 12 different longitudes in $30^{\circ}$ steps; poleward of $10^{\circ} \mathrm{S} / \mathrm{N}, 60^{\circ}$ steps were used. In this framework it was not possible to perform a thorough latitudinal investigation. Furthermore, seasonal effects were not investigated. Both will be considered in future work.

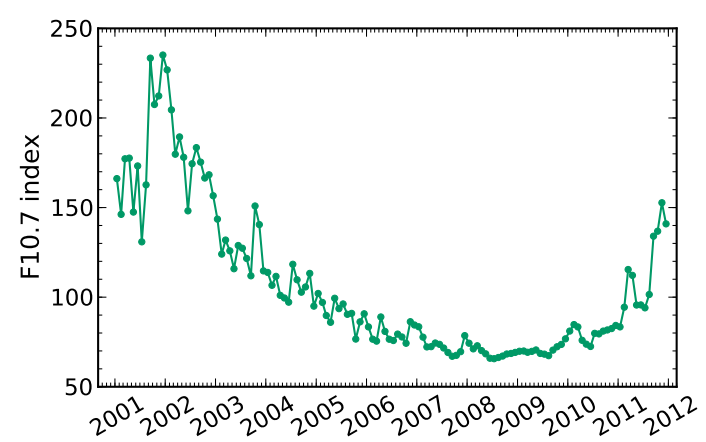

Figure 1. Monthly mean solar flux.

Furthermore, we simulated neutral atmospheric events, employing the same ECMWF analysis field from 1 January 2007, studied at the respective geographic locations - i.e., 0 , $5^{\circ} \mathrm{S} / \mathrm{N}$, and $10^{\circ} \mathrm{S} / \mathrm{N}-$ continuing in $10^{\circ}$ steps until $60^{\circ} \mathrm{S} / \mathrm{N}$, at 12 different longitudes, and above $10^{\circ} \mathrm{S} / \mathrm{N}$ simulating $60^{\circ}$ longitude steps.

In this study the ionosphere-corrected bending angle $\alpha_{C}(a)$, the L1 and L2 bending angles $\alpha_{1}(a)$ and $\alpha_{2}(a)$, and the neutral atmospheric bending angle $\alpha_{N}(a)$ were analyzed. Extremely noisy events were rejected as outliers, using a thereshold difference from the colocated neutral atmospheric bending angle of $\pm 7 \mu \mathrm{rad}$ in the altitude range 50 to $80 \mathrm{~km}$, following the suggestion of Liu et al. (2015). Random sampling showed a reduction of about $5 \%$, which is in line with results from Liu et al. (2015). Furthermore, due to large fluctuations in one of the main quantities of interest, i.e., $\left(\alpha_{C}(a)-\alpha_{N}(a)\right)$, all bending-angle profiles were vertically averaged at each impact altitude grid point over an altitude range of $5 \mathrm{~km}$, between 10 and $75 \mathrm{~km}$. The vertical averaging step smooths the bending angles and the factor $\left(\alpha_{C}(a)-\alpha_{N}(a)\right)$, without losing the information of impact altitude $a$.

Finally, the bending-angle profiles were studied as mean profiles averaged over all longitudes within a latitude band, studied separately for day and night. Additionally the following zonal climatologies were tested: $5^{\circ} \mathrm{S}$ to $5^{\circ} \mathrm{N}, 10^{\circ} \mathrm{S}$ to $10^{\circ} \mathrm{N}, 10$ to $30^{\circ} \mathrm{S}, 10$ to $30^{\circ} \mathrm{N}, 30$ to $60^{\circ} \mathrm{S}$, and 30 to $60^{\circ} \mathrm{N}$.

\section{Simulation study}

The key quantities of interest in the simulation study are the residual ionospheric error $\left(\alpha_{C}(a)-\alpha_{N}(a)\right)$, the model term $\left(\alpha_{1}(a)-\alpha_{2}(a)\right)^{2}$, and the coefficient $\kappa(a)$. In this section the temporal dependance of the first two quantities was investigated and the coefficient $\kappa(a)$ was determined from a correlation analysis between those two. Furthermore, a first test of applying the residual error model on bending-angle data was performed. 


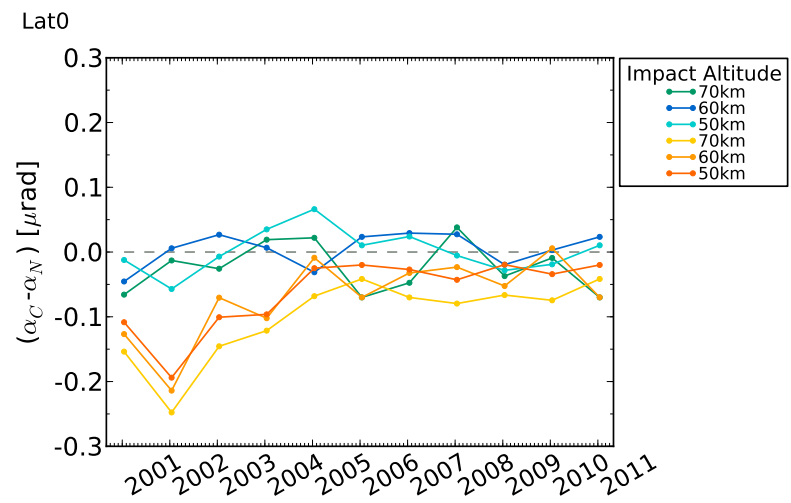

(a) Residual vs Time

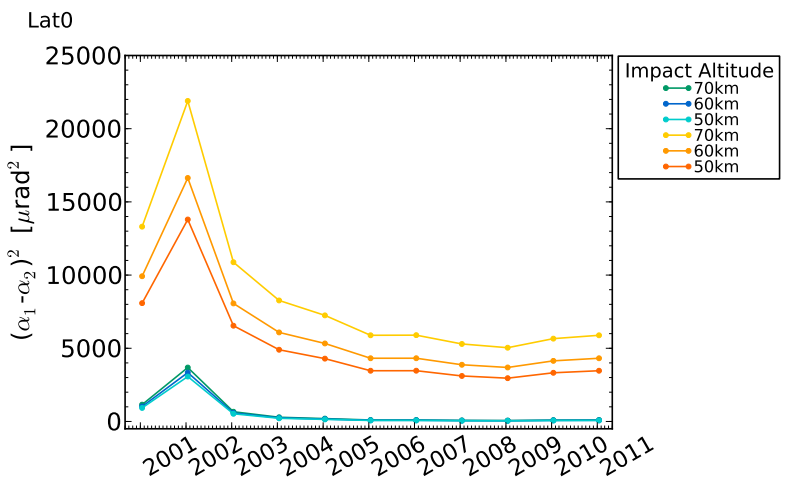

(b) Rapidly varying model term vs Time

Figure 2. Residual nighttime (blue) and daytime (orange) bending angle dependent on time (left hand side), and night- and daytime L1 and L2 bending-angle difference squared dependent on time (right hand side), studied on three impact altitudes and for latitude band $0^{\circ}$.

\subsection{Initial study of the model}

As an initial analysis we investigated for the latitude band $0^{\circ}$ the residual error and the model term as a function of time. Figure 2a shows the mean daytime and nighttime residual error, studied at different impact altitudes, while Fig. 2b shows for the same data set the model term $\left(\alpha_{1}(a)-\alpha_{2}(a)\right)^{2}$. Similar to the results of the daytime and nighttime bending-angle bias study performed by Danzer et al. (2013), we find that the nighttime residual error stays relatively constant over the period of a solar cycle, while the daytime residual error shows a clear increase in magnitude in the years of high solar activity. As one can observe, the nighttime residual ionospheric error fluctuates within the range of about $\pm 0.05 \mu \mathrm{rad}$, while the daytime residual error shows maximally an error of about $-0.25 \mu \mathrm{rad}$ at impact altitude of $70 \mathrm{~km}$ and a minimum value of about $-0.05 \mu \mathrm{rad}$.

Since the residual error is a very small number, we could observe that noise has a significant impact in the residual error analysis. The noise in the simulated data was about on the same order of magnitude as the residual error itself, which led to occasionally large fluctuations in the quantity $\left(\alpha_{C}(a)-\alpha_{N}(a)\right)$, with an even larger impact on the nighttime data due to an increased signal-to-noise ratio. The noise in the data originates due to discontinuities in the NeUoG model. While the ray tracing through the atmosphere model is really smooth and only negligible numerical noise is produced, the NeUoG ionosphere model has some discontinuities between different layers, leading to noise in the data. Furthermore, with the residual error as one of the key quantities of interest, we are studying very small numbers on the order of $10^{-7}$ to $10^{-8}$ rad. Relatively, in fractions of micro$\mathrm{rad}$, the noise from the ray tracing has a large impact on such small numbers.
To partially overcome the problem of the noise, we decided to perform a vertical smoothing step, as described in the Sect. 3, which reduced these large fluctuations and led to the illustrated results in Fig. 2a. On the other hand, the quantity studied in Fig. 2 b, i.e., $\left(\alpha_{1}(a)-\alpha_{2}(a)\right)^{2}$, shows very large values up to almost $25000 \mu \mathrm{rad}^{2}$. In that case, noise did not play a significant role, and a very stable and smooth function for night- and daytime bending-angle data was found, showing a distinct increase in times of high solar activity. Even the nighttime data showed a clear, although smaller, dependence on the solar cycle, indicating remaining ionization effects at night. Figure $2 b$ is of special interest since it shows that the model term $\left(\alpha_{1}(a)-\alpha_{2}(a)\right)^{2}$ is a function depending strongly on solar activity, i.e., the solar cycle. This further supports the hypothesis of Healy and Culverwell that the major part of the temporal dependence of the residual error is mainly captured by the model term $\left(\alpha_{1}(a)-\alpha_{2}(a)\right)^{2}$.

As a next step, we studied in Fig. 3 the correlation between the two quantities shown in Fig. 3. We could detect a clear linear dependence, and data fitting, using the method of least squares, was possible. The least-squares method was applied, using both day- and nighttime profiles together for the regression analysis (blue lines) and fitting just the day profiles (orange lines) because of the larger noise on the night profiles. The resulting fitting coefficients $\kappa(a)$ dependent on impact altitude $a$ (given in $\left[\mathrm{rad}^{-1}\right]$ ) are listed in Fig. $3^{4}$. The values for $\kappa$ are in good agreement with estimated values of $\kappa$ from calculations (Healy and Culverwell, 2015).

Finally $\kappa(a)$ was analyzed in Fig. 4 as a function of time, for three fixed impact altitudes and daytime conditions. $\kappa$ was determined by computing $-\left(\alpha_{C}-\alpha_{N}\right) /\left(\alpha_{1}-\alpha_{2}\right)^{2}$ for each year, at latitude band $0^{\circ}$. Clearly, for daytime conditions $\kappa(a)$ is between about 0 and $20 \mathrm{rad}^{-1}$ for each year, being

\footnotetext{
${ }^{4}$ Note that the values for the coefficient $\kappa$ are negative in Fig. 3 . The reason is that the minus sign from Eq. (4) has not been taken into account in this analysis.
} 


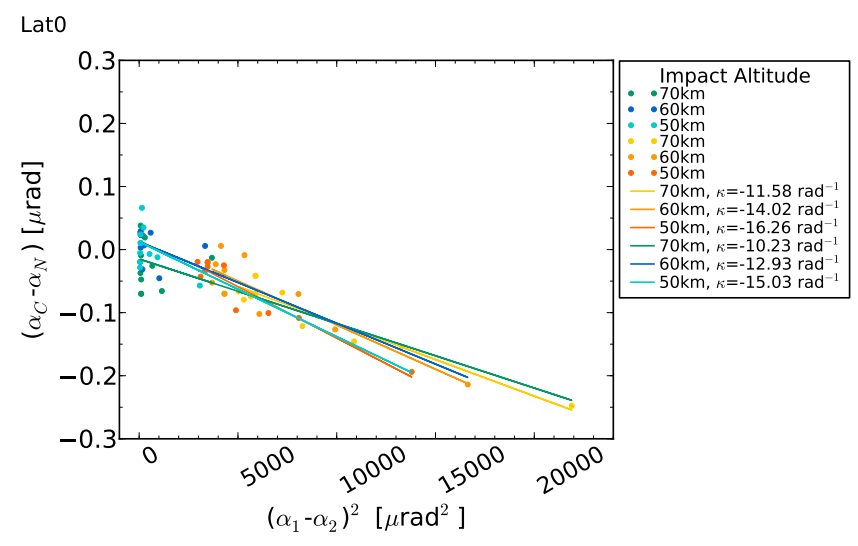

Figure 3. Scatterplot and linear fit with fitting coefficient $\kappa$ of the nighttime (blue) and daytime (orange) residual error vs. $\left(\alpha_{1}(a)-\right.$ $\left.\alpha_{2}(a)\right)^{2}$, analyzed for three impact altitudes and for latitude band $0^{\circ}$.

more or less constant with time, fluctuating mainly between about $\kappa(a)=6$ and $14 \mathrm{rad}^{-1}$. Furthermore, $\kappa(a)$ only slightly varies with altitude, showing the tendency to decrease with increasing altitude; see also fitting coefficients of Fig. 3. For nighttime conditions (not shown), the larger impact of the noise prohibited a similar analysis and $\kappa(a)$ fluctuated over a much broader range of values.

In order to perform a latitudinal investigation of the coefficient $\kappa(a)$, the same studies have been performed for other latitude bands and also for different zonal climatologies (see description of the simulated data, Sect. 3). While it was possible to study $\kappa(a)$ at low latitudes and tropical zonal bands - see the report by Danzer (2014) - it was not possible to retrieve $\kappa(a)$ at mid- to high latitudes, due to problems with noise in the simulated data. As already discussed, the small magnitude of the residual error and the magnitude of the noise are in a similar range, which led to problems of estimating $\kappa(a)$.

Nevertheless, the results shown in this section support the hypothesis that the model term $\left(\alpha_{1}-\alpha_{2}\right)^{2}$ captures the major part of the temporal variability of the residual ionospheric error, while the coefficient $\kappa(a)$ only slightly varies with altitude and shows values similar to the results found by Healy and Culverwell (2015).

\subsection{Testing the model}

In this section the proposed model for correcting the residual ionospheric error in GPS RO data is tested. As an initial investigation the correction was applied on all simulated daytime bending-angle profiles in the latitude band $0^{\circ}$, using Eq. (6) (RESIC), to retrieve the assumed neutral atmospheric bending angle.

From the one-dimensional simulation study performed by Healy and Culverwell (2015) and from first results in this study, we know that the coefficient $\kappa(a)$ varies only slightly

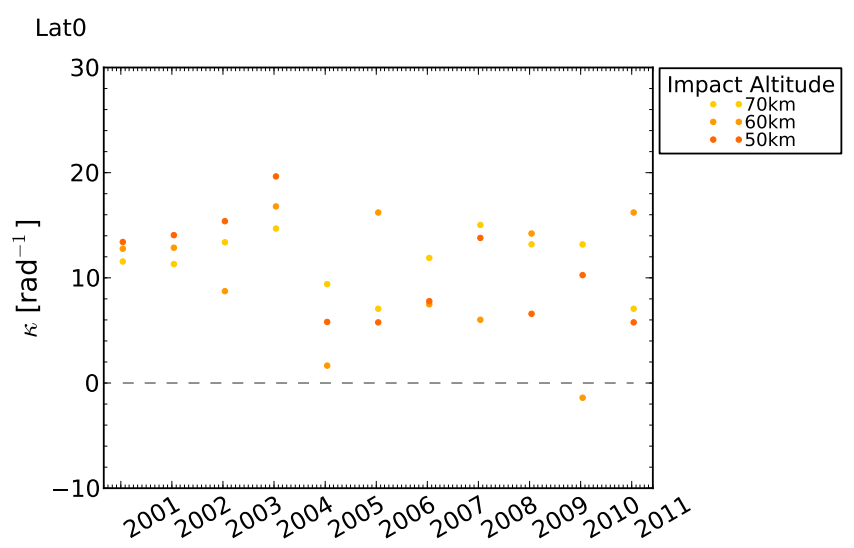

Figure 4. The coefficient $\kappa$ dependent on time for daytime profiles in the latitude band $0^{\circ}$, studied for three impact altitudes.

with impact altitude. Hence, in this first test $\kappa$ was assumed to be constant with height. We chose for the coefficient the retrieved value $\kappa=14 \mathrm{rad}^{-1}$ from simulations (see Figs. 3 and 4) and also, as a comparison, an arbitrary and larger value, $\kappa=30 \mathrm{rad}^{-1}$. Figure 5 shows the simulated residual error $\left(\alpha_{C}(a)-\alpha_{N}(a)\right)$ (orange line) in the latitude band $0^{\circ}$ dependent on impact altitude, separately for each year from 2001 until 2011. The blue line shows the calculated residual error from the model, i.e., $-\kappa\left(\alpha_{1}(a)-\alpha_{2}(a)\right)^{2}$, using $\kappa=14 \mathrm{rad}^{-1}$, while the green line shows the results when using $\kappa=30 \mathrm{rad}^{-1}$. Figure 5 shows the increased residual error in the years of high solar activity (2001 and 2002, orange line) and the decreased residual error in the minimum years (2007 and 2008, orange line). Furthermore, the plot demonstrates that the residual error computed with RESIC very well matches the residual error studied as a difference between profiles simulated with ionosphere and neutral atmospheric profiles, when using for $\kappa$ the retrieved value $\kappa=14 \mathrm{rad}^{-1}$. The RESIC model captures the temporal variability of the residual error; i.e., the model increases and decreases according to solar activity. However, when using an arbitrary value of $\kappa$, a wrong magnitude of the residual error is computed (green line). From this figure we can learn two things. On the one hand, RESIC seems to calculate the residual ionospheric error very well and the major part of the temporal variability seems to be in the $\left(\alpha_{1}(a)-\alpha_{2}(a)\right)^{2}$ factor. On the other hand, a very thorough study of the value $\kappa$ needs to be performed in order to correct for the right magnitude of the residual error.

As a next step, in Fig. 6, the impact of the residual ionospheric correction on temperatures derived from bending angles is studied. The correction was once again applied on all daytime profiles within the latitude band $0^{\circ}$, testing three values of $\kappa$ in the correction, i.e., $\kappa=10,14,20 \mathrm{rad}^{-1}$. Figure 6 shows the mean of the temperature profiles, simulated with different solar activity for each year, relative to the colocated mean of the neutral temperature profiles. The top left plot illustrates the temperature difference without a model cor- 
Lat0
$\kappa=14 \mathrm{rad}^{-1}$
$\kappa=30 \mathrm{rad}^{-1}$
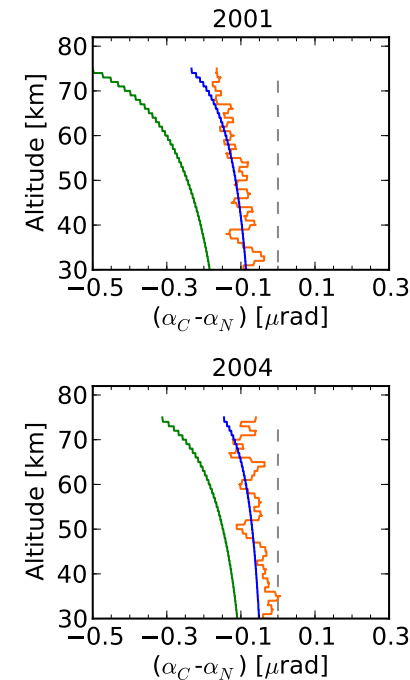

2007

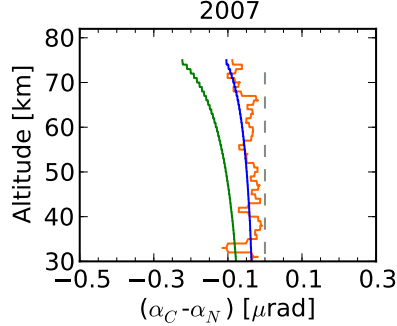

2010

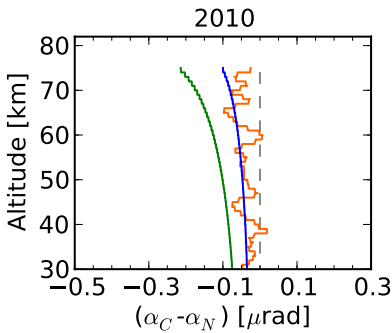

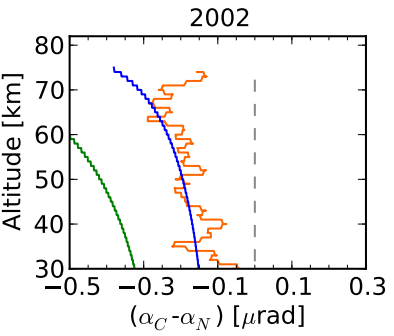
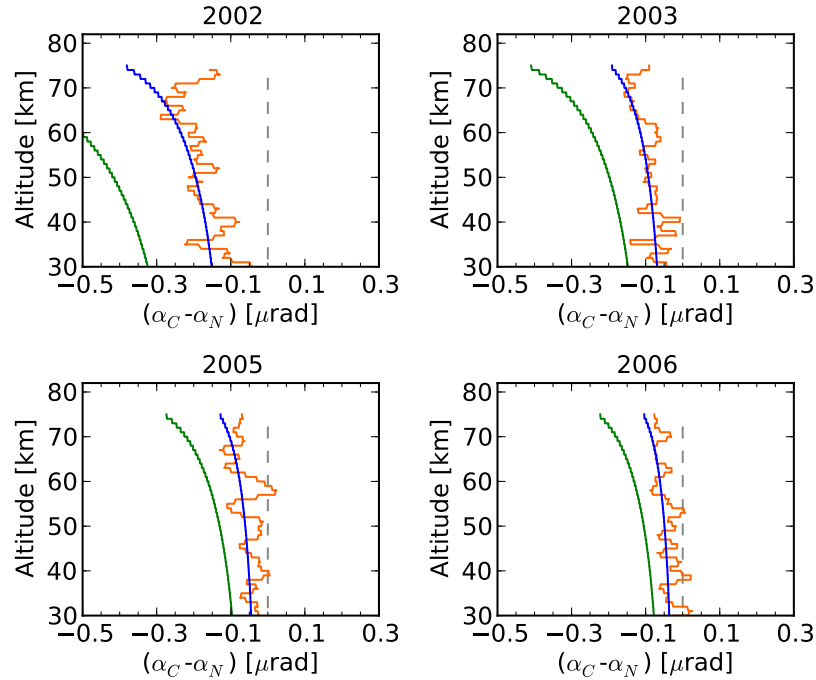

2008
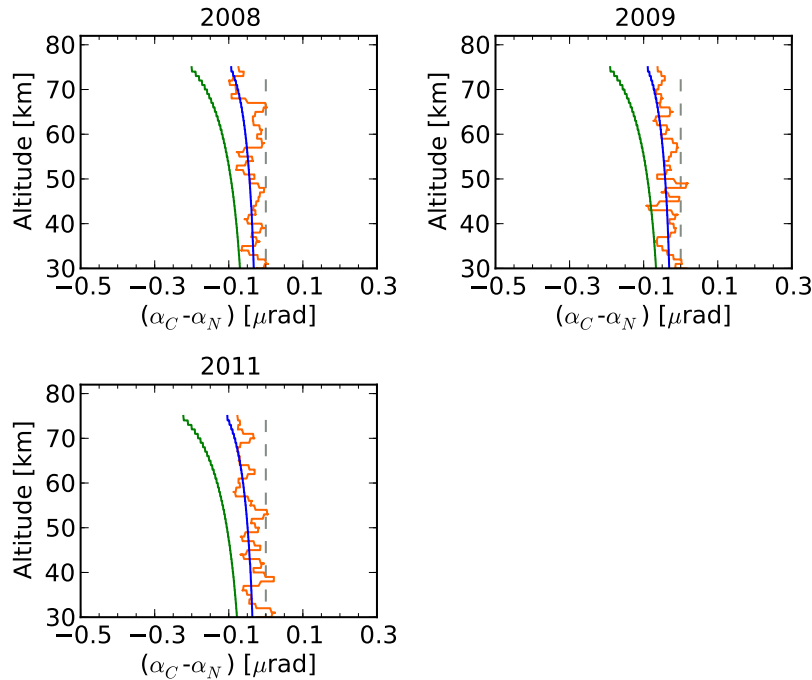

Figure 5. Comparison of the residual ionospheric correction to the monthly mean daytime residual error at latitude band $0^{\circ}$ (orange line), choosing $\kappa(a)=14 \mathrm{rad}^{-1}$ (blue) and $\kappa(a)=30 \mathrm{rad}^{-1}$ (green) in the correction model.

rection being applied, which corresponds to setting $\kappa=0$. However, the other plots show the results when RESIC is applied on the bending-angle profiles, testing the sensitivity of RESIC by using different values of $\kappa$. In the top left plot we find that profiles simulated with highest solar activity, such as 2001 and 2002, show largest temperature differences relative to the neutral atmospheric temperature profile, while towards lower solar activity, temperature differences decrease. Using $\kappa=10$ or $\kappa=14$ has a positive effect on the temperature profiles; values much closer to the neutral gas results were achieved, leading to a bunching of the profiles around 0. For example, in the high-solar-activity year 2002, the obtained simulated residual error in temperature was about $-2.2 \mathrm{~K}$ at $40 \mathrm{~km}$ altitude (top left plot), which re- duced after applying RESIC to an error of about $-0.2 \mathrm{~K}$ for $\kappa=10 \mathrm{rad}^{-1}$ (top right plot) and an error of about $0.4 \mathrm{~K}$ for $\kappa=14 \mathrm{rad}^{-1}$ (bottom left plot). The coefficient $\kappa=14 \mathrm{rad}^{-1}$ shows slightly worse results above about $40 \mathrm{~km}$ than the coefficient $\kappa=10 \mathrm{rad}^{-1}$, resulting probably due to the slowly varying latitudinal element of $\kappa(a)$; i.e., $\kappa(a)$ decreases with altitude. Using $\kappa=20 \mathrm{rad}^{-1}$ (bottom right plot) overcorrects the bending-angle data, but the distribution of profiles is still a bit narrower than setting $\kappa=0$, except for the year 2002 .

Figure 6 shows strong support for the proposed error model to be able to correct for ionospheric residuals. Furthermore, it emphasizes the importance of finding the correct value for the coefficient $\kappa(a)$. 
Temp Diff to Neutralgas Temp
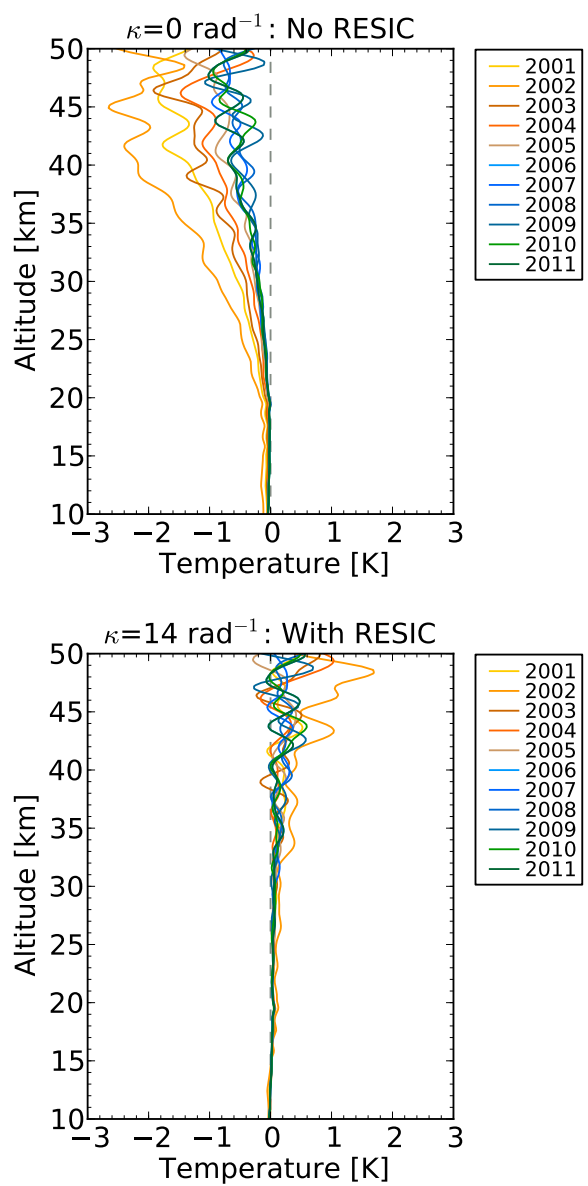

Lat0
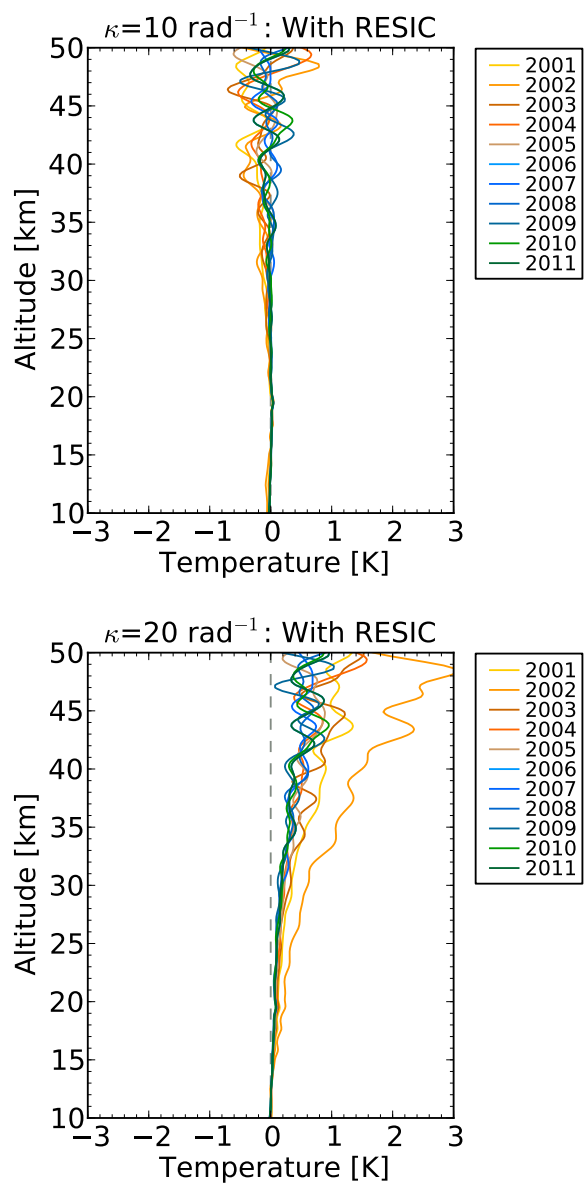

Figure 6. Testing the effect of the residual ionospheric correction on temperature profiles, studied for latitude band $0^{\circ}$ and using the coefficients $\kappa(a)=10,14,20 \mathrm{rad}^{-1}$ in the correction.

\section{Discussion and conclusions}

Residual ionospheric errors are a topic of major concern in GPS RO data. Sensitivity tests have shown that, at bendingangle level and $30 \mathrm{~km}$ altitude, residual errors of the order of about $0.05 \mu \mathrm{rad}$ add an error of about $0.5 \mathrm{~K}$ in temperature. Various first-order ionospheric corrections reduce the ionospheric error, but residual errors are still problematic (see, e.g., Mannucci et al., 2011; Danzer et al., 2013; Liu et al., 2013, 2015).

Recently, a new model for correcting the residual ionospheric error, suitable for bending-angle climatologies, was introduced and tested for simulations with a one-dimensional Chapman layer ionosphere (Healy and Culverwell, 2015). The proposed model is a product of two factors, one rapidly varying and one slowly varying. The first term depends on the solar activity - i.e., the state of the ionosphere - increasing and decreasing with the solar cycle, while the second term models the weak variation in altitude of the residual error, varying also weakly with season and geographic location.
This study was a follow-up investigation which explored the proposed model for a more complex simulation with a three-dimensional ionosphere. The simulation study enabled the investigation of the residual ionospheric error directly as the difference between bending angle climatologies simulated with ionosphere and their colocated neutral atmospheric bending-angle climatologies. Furthermore, the residual error was computed from the new residual ionospheric error model. One of the main goals was to check the hypothesis that the rapidly varying factor of the model, which depends only on measurable quantities, captures the temporal behavior of the residual ionospheric error.

It was possible to show at low latitudes correlation between the residual ionospheric error and the model term which depends on the solar activity. However, at mid- to high latitudes a decreasing signal-to-noise ratio in the simulated data prohibited us from studying correlations. From the correlation study, the model coefficient could be calculated at low latitudes, showing to be between about $\kappa(a)=6$ 
and $14 \mathrm{rad}^{-1}$. Furthermore, the model coefficient was found to be only a slowly varying function with impact altitude.

As a next step, a first attempt of correcting bending-angle data with the proposed error model was conducted. Tested for the latitude band $0^{\circ}$ and studied at temperature level, the error in temperature clearly reduced after applying the ionospheric model correction (RESIC), giving strong support for the new model.

For a thorough study of the latitudinal dependance of the coefficient $\kappa$ we suggest repeating the simulation study and producing a larger ensemble, in order to reduce noise.

Furthermore, we also propose studying the RESIC method in simulations using different models for the complex ionosphere than the NeUoG model, in order to obtain a good estimate for the coefficient $\kappa$. Another idea is to study the residual ionospheric error by analyzing GPS RO bending-angle climatologies against colocated MIPAS (Michaelson Interferometer for Passive Atmospheric Sounding) and SABER (Sounding of the Atmosphere using Broadband Emission Radiometry) bending-angle climatologies, which provide highquality data up to altitudes of $80 \mathrm{~km}$ (e.g., Remsberg et al., 2008; García-Comas et al., 2011). Performing a similar analysis as in the simulation study might also allow the retrieval of the model coefficient. The results can be compared to simulation results.

In summary, this simulation study presented some encouraging first results which support the recently proposed residual ionospheric error model. The RESIC model showed clear correlation with the simulated residual error. Furthermore we could determine the model coefficient at low latitudes, showing it to be in line with results from spherically symmetric Chapman layer ionosphere simulations. We suggest that further investigation of the proposed model for residual ionospheric correction, especially at higher latitudes, is worth undertaking.

Acknowledgements. This work was conducted as part of the Radio Occultation Meteorology Satellite Applications Facility (ROM SAF), which is a decentralized operational radio occultation processing center under EUMETSAT. J. Danzer was a ROM SAF visiting scientist for this project, and S. Healy and I. Culverwell are members of the ROM SAF. Furthermore, we thank the Wegener Center, which provided the processing and simulation software, and especially Gottfried Kirchengast, for his support and the discussions about the ionosphere model. Finally, we acknowledge the BENCHCLIM project (P22293-N21), which provided the basis for this cooperation.

Edited by: J. Y. Liu

\section{References}

Ao, C. O., Mannucci, A. J., and Kursinski, E. R.: Improving GPS radio occultation stratospheric refractivity retrievals for climate benchmarking, Geophys. Res. Lett., 39, L12701, doi:10.1029/2012GL051720, 2012.

Cardinali, C. and Healy, S.: Impact of GPS radio occultation measurements in the ECMWF system using adjointbased diagnostics, Q. J. Roy. Meteor. Soc., 140, 2315-2320, doi:10.1002/qj.2300, 2014.

Danzer, J.: Testing a new model for the residual ionospheric correction error in GPS radio occultation retrievals, Tech. rep., ROM SAF, CDOP-2 Visiting Scientist Report 23, Ref: SAF/ROM/DMI/REP/VS/24, available at: http://www.romsaf. org, last access: September 2014

Danzer, J., Scherllin-Pirscher, B., and Foelsche, U.: Systematic residual ionospheric errors in radio occultation data and a potential way to minimize them, Atmos. Meas. Tech., 6, 2169-2179, doi:10.5194/amt-6-2169-2013, 2013.

Danzer, J., Gleisner, H., and Healy, S. B.: CHAMP climate data based on the inversion of monthly average bending angles, Atmos. Meas. Tech., 7, 4071-4079, doi:10.5194/amt-7-4071-2014, 2014.

Foelsche, U., Pirscher, B., Borsche, M., Kirchengast, G., and Wickert, J.: Assessing the climate monitoring utility of radio occultation data: from CHAMP to FORMOSAT3/COSMIC, Terr. Atmos. Ocean. Sci., 20, 155-170, doi:10.3319/TAO.2008.01.14.01(F3C), 2009

Fritzer, J., Kirchengast, G., and Pock, M.: End-to-End Generic Occultation Performance Simulation and Processing System Version 5.5 (EGOPS5.5) Software User Manual, Tech. rep., WEGC and IGAM, WEGC-EGOPS-2009-TR01, University of Graz, Graz, Austria, 504 pp., 2009.

García-Comas, M., Funke, B., López-Puertas, M., BermejoPantaleón, D., Glatthor, N., von Clarmann, T., Stiller, G., Grabowski, U., Boone, C. D., French, W. J. R., Leblanc, T., López-González, M. J., and Schwartz, M. J.: On the quality of MIPAS kinetic temperature in the middle atmosphere, Atmos. Chem. Phys., 12, 6009-6039, doi:10.5194/acp-12-6009-2012, 2012.

Gleisner, H. and Healy, S. B.: A simplified approach for generating GNSS radio occultation refractivity climatologies, Atmos. Meas. Tech., 6, 121-129, doi:10.5194/amt-6-121-2013, 2013.

Hajj, G. A., Kursinski, E. R., Romans, L. J., Bertiger, W. I., and Leroy, S. S.: A technical description of atmospheric sounding by GPS occultation, J. Atmos. Sol.-Terr. Phy., 64, 451-469, doi:10.1016/S1364-6826(01)00114-6, 2002.

Healy, S. B. and Culverwell, I. D.: A modification to the standard ionospheric correction method used in GPS radio occultation, Atmos. Meas. Tech., 8, 3385-3393, doi:10.5194/amt-83385-2015, 2015.

Healy, S. B. and Thépaut, J. N.: Assimilation experiments with CHAMP GPS radio occultation measurements, Q. J. Roy. Meteor. Soc., 132, 605-623, doi:10.1256/qj.04.182, 2006.

Ho, S.-P., Hunt, D., Steiner, A. K., Mannucci, A. J., Kirchengast, G., Gleisner, H., Heise, S., von Engeln, A., Marquardt, C., Sokolovskiy, S., Schreiner, W., Scherllin-Pirscher, B., Ao, C., Wickert, J., Syndergaard, S., Lauritsen, K. B., Leroy, S., Kursinski, E. R., Kuo, Y.-H., Foelsche, U., Schmidt, T., and Gorbunov, M.: Reproducibility of GPS radio occultation data for cli- 
mate monitoring: profile-to-profile inter-comparison of CHAMP climate records 2002 to 2008 from six data centers, J. Geophys. Res., 117, D18111, doi:10.1029/2012JD017665, 2012.

Kursinski, E. R., Hajj, G. A., Schofield, J. T., Linfield, R. P., and Hardy, K. R.: Observing Earth's atmosphere with radio occultation measurements using the Global Positioning System, J. Geophys. Res., 102, 23429-23465, doi:10.1029/97JD01569, 1997.

Ladreiter, H. P. and Kirchengast, G.: GPS/GLONASS sensing of the neutral atmosphere: model-independent correction of ionospheric influences, Radio Sci., 31, 877-891, doi:10.1029/96RS01094, 1996.

Leitinger, R. and Kirchengast, G.: Easy to use global and regional models - a report on approaches used in Graz, Acta Geod. Geophys. Hu., 32, 329-342, 1997.

Leitinger, R., Titheridge, J. E., Kirchengast, G., and Rothleitner, W.: A "simple" global empirical model for the F layer of the ionosphere, Tech. rep., University Graz, Graz, 697-704, 1995.

Liu, C., Kirchengast, G., Zhang, K., Norman, R., Li, Y., Zhang, S., Carter, B., Fritzer, J., Schwaerz, M., Choy, S., Wu, S. Q., and Tan, Z. X.: Characterisation of residual ionospheric errors in bending angles using GNSS RO end-to-end simulations, Adv. Space Res., 52, 821-836, 2013.

Liu, C. L., Kirchengast, G., Zhang, K., Norman, R., Li, Y., Zhang, S. C., Fritzer, J., Schwaerz, M., Wu, S. Q., and Tan, Z. X.: Quantifying residual ionospheric errors in GNSS radio occultation bending angles based on ensembles of profiles from endto-end simulations, Atmos. Meas. Tech. Discuss., 8, 759-809, doi:10.5194/amtd-8-759-2015, 2015.

Mannucci, A. J., Ao, C. O., Pi, X., and Iijima, B. A.: The impact of large scale ionospheric structure on radio occultation retrievals, Atmos. Meas. Tech., 4, 2837-2850, doi:10.5194/amt-42837-2011, 2011.

NOAA: $F_{10.7}$ data, available at: http://ftp.ngdc.noaa.gov/STP/ SOLAR_DATA/SOLAR_RADIO/FLUX/Penticton_Observed/ daily/DAILYPLT.OBS, last access: March 2012.

Remsberg, E., Marshall, B., Garcia-Comas, M., Krueger, D., Lingenfelser, G., Martin-Torres, J., Mlynczak, M., Russell, J., Smith, A., Zhao, Y., Brown, C., Gordley, L. L., Lopez-Gonzalez, M. J., Lopez-Puertas, M., She, C.-Y., Taylor, M. J., and Thompson, R. E.: Assessment of the quality of the Version 1.07 temperature-versus-pressure profiles of the middle atmosphere from TIMED/SABER, J. Geophys. Res.-Atmos., 113, D17101, doi:10.1029/2008JD010013, 2008.
Rocken, C., Schreiner, B., Sokolovskiy, S., Hunt, D., and Syndergaard, S.: Formosat-3/COSMIC, The Ionosphere as Signal and Noise, Space Weather Workshop, Boulder, CO, USA, 2008.

Rocken, C., Schreiner, W., Sokolovskiy, S., and Hunt, D.: Ionospheric errors in COSMIC radio occultation data, paper presented at the 89th American Meteorological Society Annual Meeting, 89th American Meteorological Society Annual Meeting, Phoenix, AZ, USA, 2009.

Schreiner, B., Sokolovskiy, S., Hunt, D., Ho, B., and Kuo, B.: Use of GNSS radio occultation data for climate applications, presentation at the World Climate Research Programme (WCRP) workshop, Denver, CO, USA, 2011.

Spilker, J. J.: Signal structure and performance characteristics, in: Global Positioning System, edited by: Janiczek, P. M., papers published in Navigation, 1, Institute of Navigation, Washington, DC, USA, Berlin, New York, 29-54, 1980.

Steiner, A. K., Kirchengast, G., Foelsche, U., Kornblueh, L., Manzini, E., and Bengtsson, L.: GNSS occultation sounding for climate monitoring, Phys. Chem. Earth Pt. A, 26, D09102, doi:10.1016/S1464-1895(01)00034-5, 2001.

Steiner, A. K., Hunt, D., Ho, S.-P., Kirchengast, G., Mannucci, A. J., Scherllin-Pirscher, B., Gleisner, H., von Engeln, A., Schmidt, T., Ao, C., Leroy, S. S., Kursinski, E. R., Foelsche, U., Gorbunov, M., Heise, S., Kuo, Y.-H., Lauritsen, K. B., Marquardt, C., Rocken, C., Schreiner, W., Sokolovskiy, S., Syndergaard, S., and Wickert, J.: Quantification of structural uncertainty in climate data records from GPS radio occultation, Atmos. Chem Phys., 13, 1469-1484, doi:10.5194/acp-13-1469-2013, 2013.

Syndergaard, S.: On the ionosphere calibration in GPS radio occultation measurements, Radio Sci., 35, 865-883, 2000.

Vorob'ev, V. V. and Krasil'nikova, T. G.: Estimation of the accuracy of the atmospheric refractive index recovery from Doppler shift measurements at frequencies used in the NAVSTAR system, Izv. Atmos. Ocean. Phys., 29, 602-609, 1994. 